\title{
Informetria: explorando bases de dados como instrumentos de análise
}

Irene Wormell

\section{Resumo}

O artigo aborda os programas de pesquisa do Centro de Estudos Informétricos de Copenhague. Descrevendo a informetria como subcampo da bibliometria, discute uma nova abordagem para a área, qual seja, a combinação de teorias e metodologias avançadas de recuperação da informação com o estudo científico dos fluxos da informação. O Centro objetiva aplicar métodos bibliométricos não somente em estudos cienciométricos e em avaliações da pesquisa científica e tecnológica, mas também na análise de suas relações sociais, econômicas etc., ampliando as análises bibliométricas tradicionais para abranger as comunidades não acadêmicas nas quais a informação é produzida, comunicada e usada. A autora também convoca os profissionais de biblioteconomia e ciência da informação para enfrentarem o desafio dessa nova área de estudos quantitativos, aprendendo a explorar as bases de dados também como um instrumento para desenvolver atividades de análise, enfatizando as possibilidades que esses profissionais têm para elevar o nível de suas posições hierárquicas, assim como para explorar as técnicas informétricas no gerenciamento de políticas e de tomadas de decisão.

\section{Palavras-chave}

Bibliometria; Informetria.

\section{INTRODUÇÃO}

O objetivo deste artigo é chamar a atenção para as muitas e novas possibilidades que a análise informétrica oferece hoje para os que desejam explorar as bases de dados como um arquivo e como um instrumento de análise, quer dizer, é preciso aprender a explorar bases de dados on-line não somente para ter acesso a documentos ou a fatos, mas também para traçar as tendências e o desenvolvimento da sociedade, das disciplinas científicas e das áreas de produção e consumo. Esse tipo de informação encontrado em bases de dados, entretanto, é visível somente para o pesquisador perspicaz e para aqueles que aprenderam a "ler nas entrelinhas" da informação eletrônica.

As modernas técnicas de recuperação da informação oferecem toda uma série de facilidades que agregam novos valores para as buscas on-line na forma de análises quantitativas. É um grande desafio para os que desejam desenvolver suas próprias posições e suas especialidades, alcançando rapidamente escalões superiores na hierarquia da biblioteconomia e da ciência da informação.

A bibliometria é comumente associada à medida quantitativa de documentos. Para a maioria dos profissionais da informação, hoje, ela parece ser a parte tradicional e menos interessante da atividade documentária no meio acadêmico e, com poucas exceções, é negligenciada pela maioria dos programas curriculares atuais. Ainda não se compreendeu adequadamente que os mecanismos avançados de busca on-line e as técnicas de recuperação da informação aumentaram de forma considerável as potencialidades da metodologia de estudos bibliométricos para recuperar informação analisada a partir de grandes coleções de dados bibliográficos.
A informetria é um subcampo emergente da ciência da informação, baseada na combinação de técnicas avançadas de recuperação da informação com estudos quantitativos dos fluxos da informação.

\section{BIBLIOMETRIA OU INFORMETRIA}

A bibliometria se refere a uma variedade de regularidades tomadas de diferentes campos, exibindo uma variedade de formas. Embora as distribuições bibliométricas sejam muito diferentes em sua aparência, elas podem ser pensadas como versões de uma única regularidade, de modo que podemos falar em leis bibliométricas e suas manifestações. A Lei de dispersão de Bradford, a Lei de Zipf e a Lei de Lotka são as mais conhecidas, tratando de fenômenos importantes ou de "regularidades" encontradas na comunicação científica.

Em 1954, quando Eugene Garfield propôs a criação de índices de citação, seu objetivo básico era melhorar a recuperação da informação científica e introduzir uma forma alternativa para analisar artigos científicos, evitando as formas de representação baseadas na lingüística e na indexação. Naquela época, havia insatisfação generalizada com os serviços de indexação e resumo organizados a partir das disciplinas tradicionais. Todos eram publicados com excesso de atraso, a indexação era inconsistente e descoordenada, as políticas de seleção deixavam grandes falhas na cobertura.

Entretanto, revendo os 40 anos de história das bases de dados de citação do Institute for Scientific Information (ISI), podemos afirmar que a principal vantagem da indexação de citações - sua capacidade de transpor o uso de formas lingüísticas comuns como palavras do título, palavras-chave ou cabeçalhos de assunto - ainda não foi percebida pela 
maior parte dos profissionais da informação. O papel simbólico representado pela citação na representação do conteúdo de documentos é uma dimensão ampla da recuperação da informação: em combinação com várias expressões lingüísticas naturais, os índices de citação podem melhorar consideravelmente as buscas exaustivas de literatura.

Dessa forma, os índices de citação têm sido usados basicamente com propósito distinto do pretendido por seu criador. A idéia dos padrões de freqüências de citação (isto é, o fator de impacto das revistas), hoje amplamente empregada para estudos de avaliação, tornouse a mais utilizada medida derivada da análise de citação, recebendo muito mais atenção do que o uso originalmente proposto para os índices de citação, qual seja, recuperar informações.

Desde o começo da década de 80, a bibliometria tem evoluído para uma disciplina científica distinta com vários subcampos e estruturas de comunicação científica correspondentes. (Scientometrics, a primeira revista internacional especializada na área, é publicada desde 1979; as conferências internacionais começaram a ser organizadas em 1983.)

Os conceitos individuais dos subcampos da bibliometria, informetria, cienciometria e tecnometria não são, infelizmente, muito claros, existindo um caos terminológico na área. Na conferência internacional de 1987, foram apresentadas algumas sugestões para mudar o nome da disciplina para informetria e, desde o final da década de 80, evidencia-se uma preferência pelo uso desse termo. Porém, paralelamente a isso, tanto a bibliometria como a cienciometria também são termos empregados com freqüência. $O$ campo está se transformando em uma disciplina científica, incluindo todos os aspectos estatísticos e matemáticos relacionados aos problemas da biblioteconomia, da documentação e da informação, com fortes vínculos com os aspectos teóricos da recuperação da informação.
Atualmente, o campo da bibliometria como um todo inclui todos os aspectos quantitativos e os modelos da comunicação científica e do armazenamento, disseminação e recuperação da informação científica. Esse conceito de bibliometria é muito mais amplo que as definições usuais do termo e objetiva incorporar todas as orientações correntes, como suas aplicações à política científica, à biblioteconomia e à recuperação da informação.

No estágio em que se encontra o atual desenvolvimento profissional, um grande desafio se coloca para os profissionais da biblioteconomia e da ciência da informação em todo o mundo, no sentido de aperfeiçoar suas habilidades analíticas e agregar novos valores a seus serviços, com o auxílio de avançadas técnicas de busca on-line.

Devido ao uso freqüente e também impróprio da análise de citação para a avaliação de desempenho de pesquisas individuais, os profissionais da informação geralmente mostram pouco interesse nas implicações que a análise quantitativa pode trazer para seus serviços. Muita emoção surge quando se discute a análise de citação em relação à avaliação da pesquisa-um campo que sofre a falta de instrumentos adequados para uma análise objetiva. É por isso que a validação é tão importante. Ao mesmo tempo em que há extensa literatura sobre análise de citação, existem poucos estudos que poderiam ser reconhecidos como "validados", que confirmam seu valor na busca bibliográfica ou nos estudos de avaliação.

O valor sofisticado do serviço de informação on-line, hoje, está no uso de bases de dados não somente para recuperar informações, mas também para analisar/sintetizar os resultados e combiná-los com outras informações (garimpo de dados). Assim, a técnica de busca on-line deve ser compreendida como um processo que agrega valor em termos dos procedimentos de seleção e refinamento realizados com base em estratégias de busca inteligentes.

Para oferecer esse tipo de serviço, o profissional da informação precisa ter habilidades analíticas e criatividade, além de estar familiarizado com as técnicas avançadas de busca on-line.
Acredito que os modernos profissionais da informação, usando os métodos bibliométricos na exploração de bases de dados e em seu trabalho analítico, têm grande potencial para desenvolver novas posições e especialidades em seus serviços. Isso pode rapidamente conduzi-los a escalões mais elevados na hierarquia de seus ambientes de trabalho.

Sem o apoio à imagem profissional e ao status da área de biblioteconomia e ciência da informação (que ocorre às vezes em ambientes auto-referenciados), deve-se estar alerta todo o tempo para atrair a atenção para a utilidade e o valor do papel intermediário que os profissionais da informação têm no acesso e no refinamento da informação, e estar preparado para mostrar que as formas de desempenhar esse papel estão continuamente acompanhando as necessidades de seus usuários.

\section{CENTRO DE ESTUDOS INFORMÉTRICOS}

O Centro de Estudos Informétricos (CIS), criado junto à Escola Real de Biblioteconomia e Informação (Royal School of Library and Information Studies), em Copenhague, está baseado na longa tradição da Escola em conduzir estudos bibliométricos e nos resultados mais recentes de pesquisas em disciplinas como as teorias e metodologias de recuperação da informação; avaliação da pesquisa; análise de tendências; gerenciamento de decisões; negócios e inteligência social. A finalidade do CIS é funcionar como uma unidade interdisciplinar da Escola para realizar, em cooperação, pesquisas em vários campos.

Estudos informétricos expressam a nova abordagem do CIS para o estudo científico dos fluxos da informação: métodos bibliométricos aperfeiçoados são aplicados não somente aos estudos cienciométricos e às avaliações da pesquisa em ciência e tecnologia (C\&T), mas também à análise de suas mútuas relações sociais, econômicas etc. CIS indica uma abertura recente das análises bibliométricas tradicionais para abranger as comunidades não-acadêmicas nas quais a informação é produzida, comunicada e usada. 
O Centro objetiva realizar pesquisas, oferecer consultoria, desenvolver programas educacionais para os níveis de graduação, mestrado e doutorado e estabelecer relações de cooperação com organizações e redes profissionais do país e do exterior.

Considerando que as metodologias quantitativas são uma área pouco explorada atualmente nas pesquisas em biblioteconomia e ciência da informação, o Centro tem dirigido esforços para o fortalecimento das relações entre a bibliometria e a recuperação da informação, explorando os problemas básicos e as limitações inerentes à coleção de dados bibliométricos on-line e sua análise. Apesar dos obstáculos associados às bases de dados e ao processo de recuperação on-line, da indexação de assuntos deficiente, das inconsistências nas formas dos nomes de autores, instituições e revistas, da carência de dados vitais ou da sua inacessibilidade na estrutura das bases de dados, tem sido demonstrado que amplas análises bibliométricas on-line são, de muitas formas, mais fáceis, rápidas e menos dispendiosas quando realizadas localmente do que aquelas feitas usando-se versões das bases de dados em CD-ROM. Ingwersen e Hjortgaard Christensen têm discutido, freqüentemente nos últimos anos, as possibilidades e, de uma certa forma, as limitações de realizar análises on-line de publicação e de citação. Por meio de estudos de caso e exemplos, os autores oferecem uma descrição detalhada das vantagens e deficiências de vários métodos, assim como as possíveis soluções para problemas específicos (Hjortgaard Christensen e Ingwersen ${ }^{1,2}$ ).

Outra área de pesquisa relacionada às atividades do Centro é a análise dos procedimentos correntes de indexação e de recuperação, como forma de melhorar a representação do conhecimento impresso nos textos bibliográficos, bem como aperfeiçoar o acesso à informação armazenada (Ingwersen e Wormell ${ }^{3,4}$; Wormell ${ }^{5}$ ).

Nos últimos 10 anos, pesquisadores do Centro têm se envolvido em estudos de casos dinamarqueses, combinando metodologias bibliométricas e o uso de bases de dados de grande porte, visando à análise de tendências e o mapea- mento do conhecimento. Em cooperação com analistas de mercado e outros especialistas, os pesquisadores coletaram dados e informações que serviram como subsídio para importantes decisões administrativas e para a formulação de políticas (Ingwersen e Wormell ${ }^{4}$ ). Essas investigações são um exemplo dos benefícios da pesquisa interdisciplinar, em que a bibliometria, a recuperação da informação e as metodologias de administração interagiram de um modo profícuo.

Essas experiências ofereceram também a fundamentação filosófica para o CIS:

- transformar as metodologias bibliométricas em instrumentos úteis e viáveis nas mãos dos modernos profissionais da informação;

- estender as fronteiras da bibliometria para além da sua abrangência tradicional da comunicação acadêmica da informação;

- combinar a bibliometria com o uso de técnicas avançadas de recuperação da informação;

- promover o uso e a exploração de bases de dados e de redes como instrumentos de análise para a tomada de decisões "informadas" e para a formulação de políticas.

As metodologias e os estudos de caso desenvolvidos pelos pesquisadores do CIS irão, espera-se, aumentar o uso de bases de dados on-line também nas áreas em desenvolvimento da Ásia. Utilizando as metodologias clássicas da análise bibliométrica junto com os modernos mecanismos de busca on-line, os profissionais da informação podem ampliar consideravelmente os horizontes do seu trabalho: podem obter acesso não apenas a documentos completos ou a partes de documentos, mas também traçar as tendências e os desenvolvimentos na região. Por meio da análise quantitativa, podem mapear novos caminhos em áreas específicas da pesquisa, produção e consumo, ou identificar pequenas unidades de informação de grande valor em meio aos enormes volumes de informação eletrônica.
O Centro de Estudos Informétricos foi inaugurado em 15 de dezembro de 1997 com a presença do doutor Eugene Garfield, diretor emérito do ISI, cuja apresentação Dos índices de citação à informetria: é o rabo que está abanando o cachorro agora? abordou, resumidamente, os índices de citação como meio para a recuperação e a disseminação da informação e para o registro da história da ciência contemporânea. $O$ autor enfatizou que o estabelecimento do Centro indica a transformação da análise de citação no novo campo da informetria. Esse evento significa a emergência de abordagens integradas entre os subcampos da bibliometria, assim como retoma o interesse pelos estudos quantitativos nos programas dos cursos de biblioteconomia e ciência da informação.

Para ilustrar o objetivo e a natureza das análises informétricas, com base em táticas de busca adequadas e no uso inovador da combinação de informações extraídas de várias fontes, três estudos realizados recentemente pelo CIS são apresentados a seguir. Maiores informações sobre projetos, publicações, cursos, seminários e recursos informétricos podem ser encontradas no site do CIS: http://www.db.dk/cis.

\section{AS PESQUISAS DO CIS: TRÊS EXEMPLOS}

\section{Análises informétricas na World Wide Web}

Os pesquisadores do CIS têm investigado a interessante idéia de utilizar os métodos informétricos na WWW, lançando as bases de uma nova área: a "webmetria". Publicações recentes têm apresentado um método aplicável para uma análise informétrica geral na WWW, acompanhado de estudos de casos que examinaram Web sites dinamarqueses, noruegueses, suecos e internacionais e sua visibilidade relativa na Net em comparação com posições relevantes nas bases de dados científicas (Almind e Ingwersen ${ }^{6}$, Ingwersen ${ }^{7}$ ).

É preciso observar que, enquanto outros pesquisadores investigam a WWW em uma perspectiva quantitativa, o CIS estuda a idéia de realizar os mesmos tipos de análises informétricas na WWW que seja possível por meio de uma base 
de dados de citações. É óbvio que os métodos informétricos que usam a contagem de palavras podem ser aplicados aqui; o aspecto inovador é pensar na WWW como uma rede de citações, em que as unidades tradicionais de informação e as citações que se fazem delas são substituídas pelas páginas da Web. Essas páginas são as unidades de informação na Web, e seus hyperlinks atuam como citações.

Os debates sobre os elementos mais importantes da webmetria podem ser resumidos da seguinte maneira: enquanto nas bases de citações do ISI, os descritores são encontrados em três formas - autor, palavra-chave, palavraschave adicionais e frentes de pesquisa -, na Web, os descritores estão disponíveis tanto por autor como por freqüências. Para os pontos de acesso temáticos das páginas da Web, um autor pode usar tags (marcações), por exemplo, $<$ em > e <strong >. As freqüências dos termos são medidas por alguns índices da Web. Os títulos das páginas da rede são encontrados tanto na tag <TITLE> como em $<\mathrm{H} 1>$, sendo indentificados individualmente por sua URL (Universal Resource Locator - o endereço da página). Se o autor for uma pessoa ou uma entidade só pode ser identificado manualmente. Nas páginas da Web, a entidade ou a afiliação são fornecidas pela primeria parte da URL, mas a instituição que hospeda uma página da Web não está necessariamente vinculada ao autor dessa página. Os problemas e as desvantagens em usar análises informétricas na WWW são basicamente os mesmos dos índices de citação (estruturas de arquivo restritas e falhas na validade dos dados). A carência de qualquer imposição de padrões na forma e no conteúdo das páginas da Web, junto com a natureza dinâmica e de tempo real da rede, oferece tanto vantagens como desvantagens para o trabalho analítico, sendo estimulante testar como as metodologias de busca tradicionais funcionam nessa nova plataforma.

Em 1996 e 1997, Almind e Ingwersen ${ }^{6}$ testaram e descreveram o núcleo essencial dessas opções, implementando-as para esboçar um quadro do uso da WWW na Dinamarca, comparando-o com a Noruega e a Suécia e complementando-o com um panorama dos tipos de página, disciplina, extensão e número de links da Web. A webmetria pode ser usada para muitos propósitos e, no contexto do programa Sociedade da Informação 2000 na Ásia, existem enormes possibilidades no uso da WWW e da Hypertext Markup Language (HTML) como instrumentos analíticos para atividades tais como o gerenciamento de decisões, busca de talentos em negócios e avaliação da pesquisa.

\section{Frentes de pesquisa em agricultura e alimentos e identificação de mercados de exportação para a indústria de alimentos da Dinamarca}

Com o objetivo de identificar as áreas da pesquisa em agricultura e da produção de conhecimentos na Dinamarca que devem ser intensificadas para meIhorar a posição estratégica das exportações do país no mercado mundial, o Conselho de Pesquisa em Agricultura dinamarquês iniciou em 1989 amplo estudo para analisar os desenvolvimentos e as tendências na área de forma global. Além de usar os instrumentos tradicionais da pesquisa de mercado, também houve a necessidade de mapear determinadas atividades internacionais de pesquisa, para as quais foram utilizadas bases de dados bibliográficos de grande porte.

Alguns breves panoramas sobre o tema em outros países ofereceram uma orientação global sobre as frentes de pesquisa. Após discussões com especialistas e administradores, o quadro do estudo bibliométrico foi traçado. Mediante várias verificações cruzadas e análise dos resultados de seis países selecionados, o estudo bibliométrico ofereceu informações sobre as condições da pesquisa internacional em alimentos e a posição da Dinamarca. Os administradores usaram o estudo como subsídio no estabelecimento de estratégias para o desenvolvimento de produtos competitivos para exportação (Jordbrugsdirektoratet ${ }^{8}$ ).

Um novo estudo com objetivos similares foi realizado em 1996 para verificar a validade dos resultados da pesquisa anterior e para aperfeiçoar a metodologia usando os novos mecanismos de busca on-line (comando RANK) combinado ao Excel. O período examinado foi de 1984 a 1995 . Os resultados obtidos ser- viram para observar se as tendências de 1989 se mantiveram, conhecer a visibilidade da pesquisa dinamarquesa em alimentos de maneira global, identificar quais temas ou campos de pesquisa estiveram em foco na Dinamarca nos últimos 12 anos, determinar quais são as áreas fortes e fracas da pesquisa de alimentos na Dinamarca hoje, identificar quais são as áreas de pesquisa potenciais nas quais o país tem chance de alcançar resultados para apoiar o futuro da exportação. O movimento cíclico da intensidade da pesquisa em certas áreas foi uma das descobertas mais interessantes desse estudo, convidando a continuar as análises para aprofundar áreas específicas da pesquisa de alimentos em estreita cooperação com especialistas $\left(\right.$ Wormell $\left.^{9}\right)$.

\section{Diagnosticando o impacto internacional de revistas científicas}

O objetivo dessa investigação é apresentar certos métodos quantitativos para o diagnóstico de revistas científicas, na qual seus publicadores e administradores poderiam observar o status atual dessas publicações, assim como o seu desenvolvimento em um determinado período. Seu objetivo também é promover compreensão mais aprofundada do impacto das publicações acadêmicas e dar nova dimensão à avaliação e à posição das revistas científicas, compensando o uso simplista do fator de impacto elaborado pelo ISI.

Seu foco é a internacionalidade das revistas científicas medida segundo a distribuição geográfica de seus autores, citações e assinantes. Para examinar o quão "internacionais" as revistas realmente são em sua abrangência e impacto, alguns dados foram refinados para que pudessem ser úteis tanto para seus usuários como para os publicadores responderem às seguintes questões:

- A revista é um produto nacional, internacional, continental ou intercontinental?

- Qual é a origem da sua produção intelectual (a procedência dos autores que escrevem para a revista)?

- Em quais regiões se concentram seus usuários (distribuição geográfica das citações)? 
- Para onde vai o conhecimento publicado pela revista (de quais áreas vêm as citações)?

- A distribuição de usuários corresponde à distribuição de assinantes?

A distribuição geográfica de autores, citações e assinaturas é medida e apresentada na forma de diagramas. A hipótese sobre se existe uma relação entre o padrão de distribuição de usuários e de assinantes é verificada por inferência. A correlação entre esses dados pode ter muitas explicações, mas certamente pode levantar, entre os publicadores, algumas questões e idéias úteis relativas a potenciais mercados ainda não explorados.

A "exportação de conhecimento científico" veiculado pela revista também é abordada - outro indicador de impacto medindo-se o caráter interdisciplinar da publicação e de seu campo relacionado. Isso serve para observar se uma determinada revista possui força e impacto científicos para transpor as fronteiras tradicionais da comunicação científica de seu campo de conhecimento e se é capaz de atrair autores e usuários de disciplinas vizinhas, assim como de áreas mais amplas da ciência. Usar o comando RANK para relacionar as categorias de assunto para um conjunto de citações é um método fácil, porém bastante eficiente para mapear o conhecimento exportado pela revista.

Como exemplo, selecionou-se um grupo das principais revistas da área de biblioteconomia e ciência da informação que ostenta a reputação de ter um público internacional, incluindo uma revista de um campo relacionado, no caso, ciências da computação. A lista resultante é a seguinte:

1. Libri - International Journal of Libraries and Information Services;

2. Scientometrics - an international journal for all quantitative aspects of the science of science, communication in science and science policy;

3. JASIS - Journal of the American Society for Information Science

4. J Doc-Journal of Documentation;
5. IPM - Information Management \& Processing;

6. C\&RL-College \& Research Libraries;

7. Comp J - The International Journal of Computing.

Para descrever o status atual, assim como os desenvolvimentos das revistas nos últimos 10 anos, as análises estão divididas em dois períodos, 1987-1988 e 1992-1993, ambos considerando um intervalo de cinco anos para o cálculo das citações, ou seja, 1987-1991 e 1992-1996.

Os resultados dessa pesquisa indicam claramente que a análise informétrica pode coletar novas informações e conhecimentos úteis para apoiar as decisões administrativas e a formulação de políticas editoriais. O relatório 7.0 do CIS apresenta todos os diagramas relativos às sete revistas, juntamente com seus indicadores (Wormell ${ }^{10}$ ). Naturalmente, os resultados são de grande interesse sobretudo para os publicadores, editores e administradores das revistas avaliadas, mas a metodologia é aplicável em todos os tipos de revistas e campos do conhecimento. Espera-se que os profissionais da informação descubram a sua utilidade para analisar sua própria coleção de revistas ou para assessorar seus pesquisadores na seleção de revistas que possam publicar suas pesquisas, assim como para identificar assinaturas dispendiosas.

\section{Intensidade da pesquisa em um campo do conhecimento}

O diagrama a seguir mostra a produção da pesquisa na área de bibliometria em termos globais, apresentando a distribuição geográfica de publicações em seis regiões, nas três mais importantes bases de dados de citações dentro do período 1988-1997. As informações estão baseadas na localização geográfica dos autores que publicam sobre o tema e nas principais revistas científicas internacionais incluídas nos índices de citação do ISI. Estão excluídas as publicações nacionais e outras revistas mais específicas.
Usando o comando RANK nas bases de dados Dialog, uma lista oferece informações detalhadas a respeito dos países de origem das 683 publicações resultantes da pesquisa (a produção total em 1988-1997).

A partir dessa lista, é possível observar o volume dos resultados das pesquisas na área nas seis regiões do mundo: sabe-se que os Estados Unidos têm grande tradição em bibliometria, mas, atualmente, a Europa se encontra na linha de frente dos desenvolvimentos nesse campo. Também na Índia existe forte tradição em análises bibliométricas, assim como há grupos de cientistas que trabalham ativamente com estudos cienciométricos em várias instituições de pesquisa e na área de biblioteconomia e ciência da informação.

É preciso fortalecer a informetria junto aos profissionais da informação, abrindo suas mentalidades e sua compreensão para o uso que pode ser feito desse método nos modernos serviços de informação. 


\section{FIGURA 1}

Distribuição geográfica de publicações na área de bibliometria de 1988 a 1997

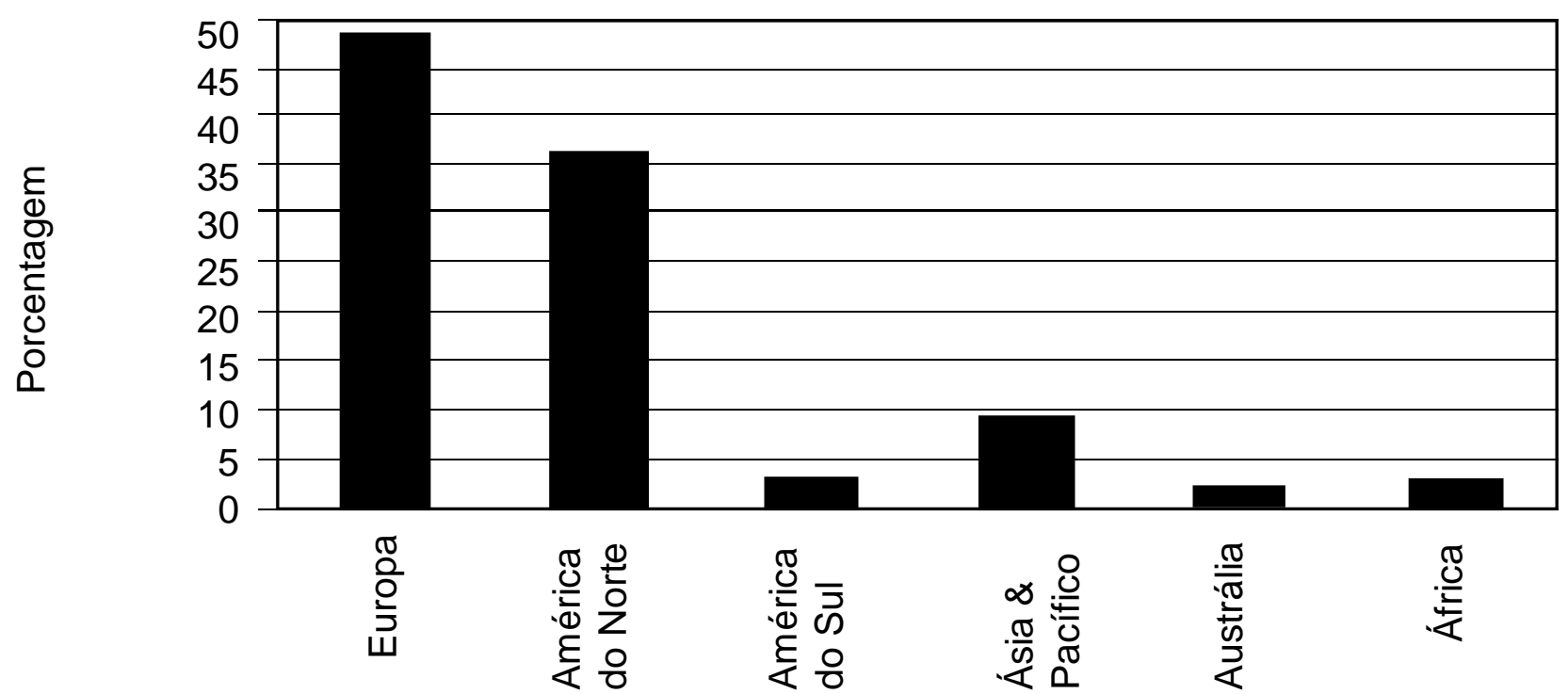

Regiões

\begin{tabular}{|c|c|c|c|c|c|c|c|}
\hline Posição & № & $\%$ & País & Posição & № & $\%$ & País \\
\hline 1 & 227 & 35.0 & EUA & 30 & 3 & 0.5 & Áustria \\
\hline 2 & 52 & 8.0 & Holanda & 31 & 3 & 0.5 & Brasil \\
\hline 3 & 37 & 5.7 & Hungria & 32 & 3 & 0.5 & Croácia \\
\hline 4 & 37 & 5.7 & Espanha & 33 & 3 & 0.5 & R. Fed. Alemã \\
\hline 5 & 36 & 5.6 & Inglaterra & 34 & 3 & 0.5 & China \\
\hline 6 & 27 & 4.2 & França & 35 & 3 & 0.5 & Taiwan \\
\hline 7 & 27 & 4.2 & Alemanha & 36 & 3 & 0.5 & Ucrânia \\
\hline 8 & 25 & 3.9 & Canadá & 37 & 3 & 0.5 & País de Gales \\
\hline 9 & 22 & 3.4 & Bélgica & 38 & 2 & 0.3 & Irã \\
\hline 10 & 21 & 3.2 & Índia & 39 & 2 & 0.3 & Cingapura \\
\hline 11 & 13 & 2.0 & Israel & 40 & 2 & 0.3 & lugoslávia \\
\hline 12 & 12 & 1.9 & USSR & 41 & 1 & 0.2 & Bielo-Rússia \\
\hline 13 & 11 & 1.7 & SSSR & 42 & 1 & 0.2 & Camarões \\
\hline 14 & 10 & 1.5 & Austrália & 43 & 1 & 0.2 & Cuba \\
\hline 15 & 9 & 1.4 & Japão & 44 & 1 & 0.2 & Rep. Tcheca \\
\hline 16 & 9 & 1.4 & África do Sul & 45 & 1 & 0.2 & Tchecoslov. \\
\hline 17 & 7 & 1.1 & Chile & 46 & 1 & 0.2 & Etiópia \\
\hline 18 & 7 & 1.1 & México & 47 & 1 & 0.2 & Grécia \\
\hline 19 & 7 & 1.1 & Rp.Pop.China & 48 & 1 & 0.2 & Hong Kong \\
\hline 20 & 7 & 1.1 & Rússia & 49 & 1 & 0.2 & Kuwait \\
\hline 21 & 6 & 0.9 & Dinamarca & 50 & 1 & 0.2 & Malásia \\
\hline 22 & 6 & 0.9 & Itália & 51 & 1 & 0.2 & Marrocos \\
\hline 23 & 6 & 0.9 & Escócia & 52 & 1 & 0.2 & Irlanda Norte \\
\hline 24 & 5 & 0.8 & Bulgária & 53 & 1 & 0.2 & Paquistão \\
\hline 25 & 5 & 0.8 & Finlândia & 54 & 1 & 0.2 & Catar \\
\hline 26 & 5 & 0.8 & Noruega & 55 & 1 & 0.2 & Arábia Saudita \\
\hline 27 & 4 & 0.6 & R.Dem. Alemã & 56 & 1 & 0.2 & Turquia \\
\hline 28 & 4 & 0.6 & Nigéria & 57 & 1 & 0.2 & Venezuela \\
\hline 29 & 4 & 0.6 & Suécia & - & & & \\
\hline
\end{tabular}




\section{REFERÊNCIAS BIBLIOGRÁFICAS}

1. HJORTGAARD CHRISTENSEN, F., INGWERSEN, P. Online citation analyses: a methodological approach. Scientometrics, v. 37 , n. 1 , p. $39-62,1996$

2. HJORTGAARD CHRISTENSEN, F., INGWERSEN, P. Data set isolation for bibliometric online analysis of research publications: fundamental methodological issues. Journal of the American Society for Information Science, v. 48, n. 3, p. 205 17, 1997.

3. INGWERSEN, P., WORMELL, I. Modern indexing and retrieval techniques matching different types of information needs; keynote address. In: FID, 1988, Helsinki. Proceedings of the $44^{\text {th }}$ FID Congress. Ed. by S. Koskiala and R. Launo. North Holland: FID, 1989. p. 79-90. (FID Publications, 675).

4. INGWERSEN, P., WORMELL, I. Databases as analytical tools in research management: a case study. In: CRONIN, B., TUDORSILOVIC, N. (eds.). The knowledge industries: levers of economic and social developments in the 1990's. London: Aslib, 1990. p. 205-16.

5. WORMELL, I. Subject access redefined: how new technology changes the concept of subject representation. In: INTERNATIONAL ISKO CONFERENCE, 1994, Copenhagen. Proceedings of the $3^{r d} \ldots$ Ed. by $\mathrm{H}$. Albrechtsen and S. Ørnager. Frankfurt: Index Verlag, 1994. p. 431-9.

\section{Informetrics: for the exploration of databases as analytical tools}

\section{Abstract}

This paper gives a sample of the research programmes of the Centre for Informetric Studies, Copenhaguen. It also describes informetrics as a sub-field of bibliometrics, discussing its new approach, i.e., the combination of advanced information retrieval theories and methodologies with the scientific study of information flows. The Centre aims to apply bibliometric methods not only to scientometric studies and research evaluations of science and technology, but also to the analysis of their societal, industrial and other special relations. This means an extension of the traditional bibliometric analyses to cover non-scholarly communities in which information is produced, communicated and used. The author also appeals to LIS professionals to face the challenge of this new area of quantitative studies, learning to explore databases also as a tool to carry out analytical work, emphasizing the possibilities for those professionals to raise their positions in the information work hierarchies, as well as to explore informetric techniques to support the management of decisions and policy making.
6. ALMIND, T., INGWERSEN, P. Informetric analyses on the World Wide Web: methodological approaches to "webmetrics". Journal of Documentation, v. 53, n. 4, p. 404-26, Sept. 1997.

7. INGWERSEN, P. The calculation of Web impact factors: research note. Journal of Documentation, v. 54, n. 1, p. 1-5, 1998.

8. JORDBRUGSDIREKTORATET. Bibliometrisk analyse af udviklingstendenser i fødevareforskning og produktion. København: Jordbrugsdirektoratet, 1989. $42 \mathrm{p}$.

9. WORMELL, I. Fødevareforskningen i Danmark, samt på Danmarks viktigste eksportmarkeder. Copenhagen: Royal School of Library and Information Studies, 1997. 33 p. (CIS Rapport, 4).

10. WORMELL, I. The international impact of scientific journals - how international are the international journals?: geographical distribution of authors, citations and subscriptions measured for seven selected LIS journals. Copenhagen: Royal School of Library and Information Studies, 1998. 40 p. (CIS Rapport, 7.0).

\section{Irene Wormell}

Ph.D, chefe do Centro de Estudos Informétricos Escola Real de Biblioteconomia e Informação, Copenhague.

IW@db.dk

Tradução do inglês de Irati Antonio

\section{Keywords}

Bibliometrics; Informetrics. 